\title{
Research on the Innovative Application Methods of Lingnan Traditional Elements in Architectural Design
}

\author{
Feifeng Zhong \\ Guangzhou Nanyang Polytechnic
}

\begin{abstract}
The focus of this study is how to break through the traditional practices, and deeply explore the innovative application methods of Lingnan traditional elements into architectural design, so that Lingnan traditional elements can be subtly integrated into modern architectural design. It is hoped that this will be of certain practical guiding significance for the architects in applying Lingnan traditional elements in architectural design.
\end{abstract}

Keywords: Lingnan traditional elements; Architectural design; Innovation; Application methods.

\section{Introduction}

The Lingnan architecture has occupied an extremely important position in the Lingnan area. It is unique in traditional Chinese architecture and has rich cultural connotations and values. However, a large part of works of Lingnan traditional elements applied in architectural design is too monotonous, symbolic, conceptualized, and even over-decorated, artificial, and formulaic. There are rare substantive studies and cases that are innovative and groundbreaking in integrating Lingnan traditional elements into modern architectural design. How to integrate Lingnan traditional elements into the architectural design ingeniously, reasonably and innovatively, so that our architectural design contains a strong Lingnan cultural heritage and typical Lingnan regional characteristics, which is an important topic in this study.

\section{Body}

The integration of Lingnan traditional elements into modern architectural design cannot be simply copied and pasted, and a variety of innovative methods should be sought. Through the study of a large amount of literature and the field investigation in Lingnan area, the author in this study believes that the innovative application methods of Lingnan traditional elements can be summarized into eleven methods. We will elaborate on these methods as follows:

\subsection{Transplantation and transformation}

According to the surrounding environment, historical culture and other factors, some elements of the traditional element in the plane or elevation modeling are directly transplanted into the architectural design and carry out certain transformation by combining with the design intent ${ }^{[1]}$. We can properly transplant Lingnan traditional figures, plants, animals, patterns with traditional metaphorical and symbolic meanings, symbols with traditional religious ornamentation, geometric symbols and other images, as well as some forms of characters and scenery in popular historical legends, myths, idioms or legends. The existing technology and design techniques can be combined to partially transform and innovate these Lingnan traditional elements, so that these Lingnan traditional elements can be naturally integrated into the architectural design, making it a new architectural expression element.

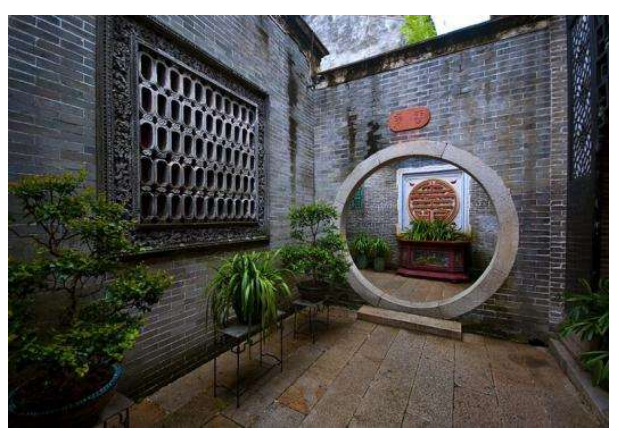

Figure 1. The brick wall with the auspicious word "Shou"in the Yuyin Garden.

For example, Wu Bin' Yuyin Garden, a private garden in the Qing Dynasty, is located in Panyu, Guangzhou. The brick wall in the Garden is decorated with the auspicious word "Shou" and then slightly modified with appropriate patterns (Fig.1), forming a Chinese character 
pattern in which the word inside, circle outside, serves as a decoration on the wall, making the originally blank wall meaningful.

\subsection{Simplification and abstraction}

The use of abstract and concise geometric patterns conforms to the principle of less being more advocated by modern design ${ }^{[2]}$. When using the Lingnan traditional elements for modern architectural design, the visual form of Lingnan traditional elements can be simplified and abstracted, so as to sort out the logical order between the elements so that these elements are directly or indirectly or figuratively or abstractly expressed and then recombined with the modern form, and then transformed into a new symbol, which makes the Lingnan traditional elements have modern form features and connotations. For example, the element of Zhuhai City Bird Seagull is extracted by the Zhuhai Nanshawan Howard Johnson Resort Hotel. The abstract and simple geometric ornaments on the glass wall outside the lobby symbolize a group of seagulls flying to the sea, reflecting the island (Fig.2). The good ecological environment also affects the enthusiasm of the guests to the sea;

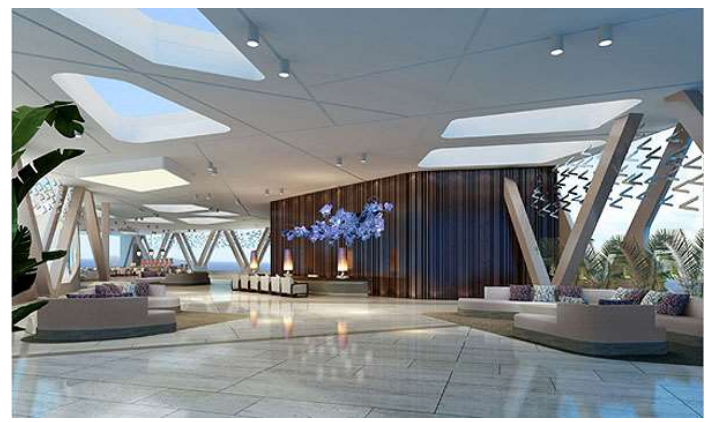

Figure 2. Hotel lobby.

For example, in the project of Zhuhai Nanshawan Howson Resort Hotel, the element of seagull, Zhuhai City bird, is extracted. The abstract and simple geometric ornaments on the glass wall outside the lobby symbolize a group of seagulls flying to the sea, reflecting the island's good ecological environment, it also affects the enthusiasm of the guests to the sea; The ceiling of the characteristic restaurant is boldly designed in the form of waves, and the free wall rises to the ceiling, which is full of great tension and dynamic.

Modern architectural design can also extract artistically beautiful graphics from Lingnan traditional paper-cutting art, and then use modern abstraction techniques to simplify the graphics and reorganize them to form architectural design works with both Lingnan traditional flavor and modern expressiveness.

\subsection{Decomposition and transformation}

The method in which one traditional element is generated into two or more other forms or elements is called decomposition. The transformation is to directly adopt the original form in the building, and transform the original traditional elements through various changing techniques.
In the process of transformation, it is needed to retain the original structure and morphology as far as possible ${ }^{[3]}$.

In modern architectural design, we should be good at fully utilizing the rule of modern formal beauty and the modern composition method, decomposing the Lingnan traditional elements, decomposing the Lingnan traditional elements into scattered fragments, forming a specious, alternative form, and then is transformed in an ideal style. The essence of Lingnan traditional culture is fully refined and transformed to form a new image, which makes the architectural design works unique and full of the historical charm of Lingnan.

This technique can be applied to many aspects of architectural design, such as the decomposition of Lingnan traditional element patterns into new patterns with simple and modern expression, the application of the eaves, walls, plinth, thresholds, interior and exterior windows, pillars and architectural sketches.

\subsection{Dispersion and reconstruction}

In the application of Lingnan traditional elements into the modern architectural design, it is needed to be good at applying modern design techniques and deconstructive means to break up and reconstruct the regional Lingnan traditional elements, so as to better reflect the regional characteristics and the connotation of Lingnan traditional elements and enhance the taste of architectural design.

On the basis of a thorough understanding of the Lingnan traditional elements, we can apply the method of deconstruction according to the needs of architectural design, apply the concept of decomposition, and reconstruct discrete and eliminate the Lingnan traditional elements by means of scattering, separation and transformation, and then use the methods of superposition, misplacement and collage to break the fixed and complete image of Lingnan traditional elements and reconstruct them into a new symbol, thus reinterpreting a new meaning of the solidified traditional Lingnan traditional elements.

We can also choose one or several typical forms from the original Lingnan traditional elements, scale, reorganize or add other new elements according to the requirements of the existing architectural design and then recombine to form a new pattern. This is also a process of breaking up and reconstructing the Lingnan traditional elements. On one hand, this method can well inherit the traditional culture and historical context, on the other hand, it can be integrated into the modern humanistic atmosphere, so that the building can show a new look without losing the traditional charm ${ }^{[4]}$.

\subsection{Exaggeration and mutation}

In the architectural design, the form of the Lingnan traditional elements will be reduced or enlarged to a certain extent, which can emphasize the structure or form of the part of the building; combined with the architectural design concepts and design ideas, some Lingnan traditional elements can be exaggerated and mutated so as to highlight the theme of architectural 
design. This is just like the exaggeration and mutation of Cantonese opera masks, each pattern can be an element in our architectural design.

For example, at the China Pavilion of the Shanghai World Expo, the Chinese character elements are exaggerated and mutated. They are deformed by the sculpt in Chinese characters and then decorated on a red wooden frame to form a practical and beautiful shading system. Here, the national traditional decoration art has formed a perfect fusion with Chinese characters. The combination of seal cutting Chinese character and red wooden frame after exaggeration and mutation gives the Chinese Pavilion a profound aesthetic meaning (Fig.3).

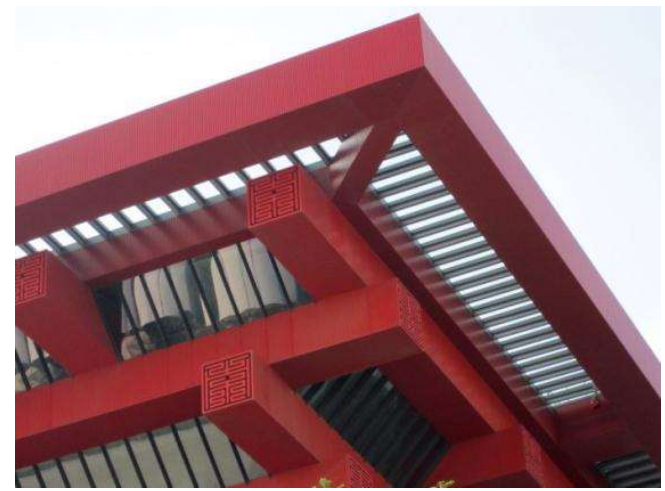

Figure 3. The seal cutting on the wooden frame of the China Pavilion at the Shanghai World Expo.

\subsection{Rhymes and rhythms}

In architectural design, rhymes and rhythms are important ways to achieve diverse unity. The rhyme is a repetitive change on the premise of unity, and the rhythm is a diverse unity based on change. The Lingnan traditional elements are incorporated into the architectural design, and the rhymes and rhythms can be formed by the density of the elements, the contrast of the volume, the change of the length of the elements, the alternating of the space and the changing colors, and the changing colors.

In the architectural design, when the same or the same set of traditional elements are repeated continuously or alternately, which can visually form a dynamic and orderly visual effect, bringing diversity to the rhythm and making it rich in rhyme beauty ${ }^{[5]}$.

In the case of the repetition of a single-modeling Lingnan traditional element, it is possible to find a breakthrough by inserting other vastly different Lingnan traditional elements, thereby producing a strong visual effect. The Lingnan traditional elements in the building are regularly repeated, and the gradual order forms a dynamic rhyme and rhythm, which will bring a new visual experience and pleasing artistic effect.

There are many ways to form the rhymes and rhythms of Lingnan traditional elements in architectural design. Common ones are simple rhyme, gradual rhyme, alternating rhyme, mimetic rhyme, and interlaced rhyme. As an architect, it is needed to be good at applying these techniques to subtly integrate the Lingnan traditional elements into the architectural roof modeling, architectural façade, architectural detail texture, interior decoration of the building in modern architectural design, giving people a refreshing feeling.

\subsection{Arrangement and combination}

In architectural design, repeated arrangement of the same element in the shape, size, color, and texture will make the building a solemn feeling; If the same element has a certain change in shape, size, color, and texture, the building will have a certain sense of order; by using the principle of mathematical arrangement and combination, some similar or approximate elements are inserted into a series of identical elements, and then different arrangement and combination are conducted to constitute multiple sets of different architectural forms; Different arrangements and combinations of different elements will result in more different architectural appearances or architectural decorative patterns; A plurality of individual elements are first combined into basic units of various shapes and then repeated by primitives. The primitives are re-arranged and combined while making some changes in size, color, virtual reality, etc., which will make the appearance of the building or the decorative patterns of the building are varied and layered (Fig.4).

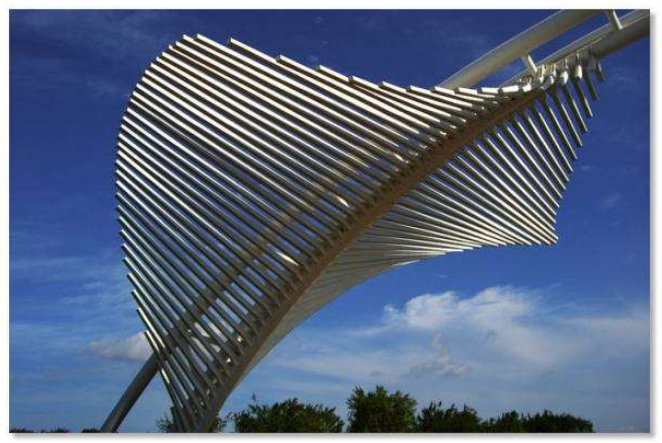

Figure 4. A rhythmic architectural sculpture.

In the "Fiveth Park" residential community of Shenzhen Vanke, in the design of the single building, the designers used the architectural symbols of Chinese traditional houses such as the floral-pendant gates of Beijing Siheyuan and the Matou Wall of Huizhou architecture, and then re-arrange and combine these traditional architectural elements, forming a new Chinese architectural style that breaks the limitations of time and space, this technique is worth learning.

\subsection{The change of light and shadow, virtual and reality}

In the application of Lingnan traditional elements in architectural design, we can not only see the actual existence of the entity but ignore the "virtual form". We can learn from the virtual-reality technique of traditional Chinese paper-cutting art and the combine the change of light and shadow to carry out architectural design. For example, there is a hard building made of metal material in Suzhou Moonlight Pier (Fig.5). The traditional elements square and circle of Chinese elements are used here. The whole building is composed of circles and 
squares of different sizes to form a transparent hollow frame. During the day, the ground forms a mottled shadow under the illumination of the sun. At night, the light and shadow change under the illumination of the light, dazzling and full of mystery ${ }^{[7]}$. This kind of innovation is achieved by applying the form of traditional elements to produce the effect of light and shadow, to achieve the effect of virtual reality and prominent theme.

Another example is the lotus leaf theme structure of Hengshui Lake International Marathon Square, which combined the lotus leaf modeling with the traditional Chinese paper-cutting art. The bright red "umbrella" top is made into a hollow modeling, casting a mottled shadow under the sun. With the orientation of the sun throughout the day, the light and shadow in different angles and directions are changed. At night, under the illumination of the surrounding garden lights and the reflection of the self-reflecting materials, the light and shadow change, with an infinite meaning (Fig.6).

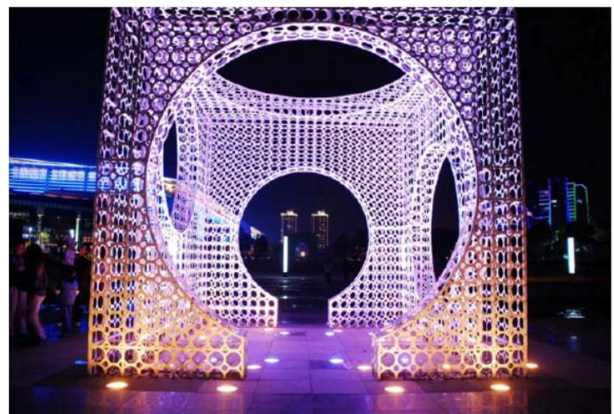

Figure 5. Metal building at Suzhou Moonlight Pier.

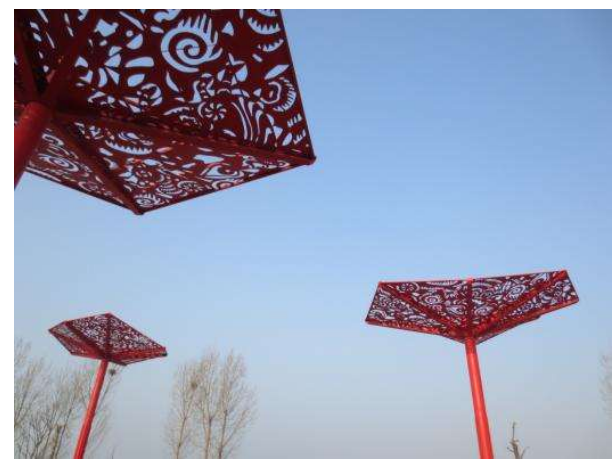

Figure 6. Lotus leaf theme structure.

\subsection{Cutting and dislocation}

The malleabl part of the same Lingnan traditional elements is cut, and then re-align, overlap, intersect, concatenate and innovate them according to the principles of composition and design concept, so that it can form new forms and bring people a new visual impact, which not only can fully reflect the connotation of the traditional Lingnan elements, but also preserve the charm of traditional art. Or to cut some representative parts of the traditional Lingnan elements, and then dislocate, overlap, reset, reconstitute and create them according to the design composition, which can produce refreshing elements.

We can learn from this technique in architectural design, such as extracting the traditional Cantonese opera mask patterns, applying cutting, isomorphism, overlapping, misalignment, intersection, juxtaposition and other methods to innovate, and then applying numerical control technology to perform precise cuts on a "rusted steel plate" with a modern atmosphere, forming a new architectural form that combines technology and art, tradition and modernity.

For example, the sculpture of the source of the folding rhyme in the architectural design of the origami museum square of the art design students of the Northwest A\&F University: on the rotating three-dimensional square entity, the shape of the four-sided windmill is shaped by cutting, folding, curling and weaving to form a hollow, dynamic art form.

\subsection{New materials and new processes}

With the increasing development of technology, new materials and processes are available for modern architectural design. Such as, luminescent fiber materials, space panels, ETFE (ethylene-tetrafluoroethylene copolymer) film, glass fiber reinforced materials, Corian, fiber plastic polymer materials, optimized honeycomb topology materials, nano materials, ceramic permeable bricks, polyester, light-sensitive materials, heat-sensitive materials, and sound control materials and other new materials, all mentioned above have very high utilization value. The introduction of these advanced technologies and processes can better integrate the Lingnan traditional elements into architectural design.

In applying Lingnan traditional elements into architectural design, if modern new materials and new techniques are combined, we can not only retain the traditional charm, but also add the beauty of modern craftsmanship, thus achieving the perfect integration of ancient and modern. For example, the water molecule structure is densely arranged on the surface of the Water Cube, which is expressed by the advanced tetrafluoroethylene materials. It is formed into a thin sheet by extrusion to form the epidermis. The outer skin structure of the Water Cube is the structure of the inflatable membrane ${ }^{[8]}$.

We can also combine traditional materials and modern materials to create a new Chinese modern architecture. For example, the application of traditional materials (such as wood, bamboo, beams, tiles, white walls) combined with modern reinforced concrete structures, then be refined and reshaped in the traditional architectural design language, which can also form buildings with unique characteristics.

For example, Ziyun Building and Xiandamen Entrance Building of Xi'an Datang Furong Garden use gold-plated metal edging on the roof and replace the paint on traditional building components with modern painting techniques, so that the color of the building remains unchanged (Fig.7), the components such as buckets, arches, and lifts all use modern concrete antique components ${ }^{[9]}$. This approach is a perfect combination of traditional elements and modern materials and modern craftsmanship, it is worthy of our application in architectural design. 


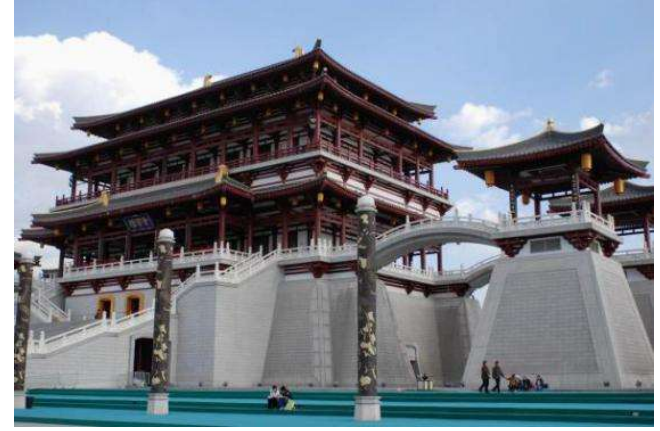

Figure 7. Ziyun Building, Xi'an Datang Furong Garden.

\subsection{Cultural blending}

In addition to reflecting the local design elements in Lingnan traditional elements and innovative application of Lingnan traditional elements, it also needs to add new content and new models in the process of inheritance in our modern architectural design, be good at absorbing and integrating foreign cultures, and fully realize the importance of the combination of Chinese and foreign elements and cultural blending ${ }^{[6]}$.

In the innovation of Lingnan traditional elements, we must be good at absorbing the excellent culture of the West, combine Chinese and Western, and be tolerant; open-minded. Only by combining Lingnan traditional elements with modern and advanced design concepts and ideas can we enrich the forms of Lingnan traditional elements and create architectural works that contain Lingnan traditional elements without losing the modern sense, making the architectural design unique.

First of all, it is needed to study and deeply understand Lingnan traditional cultural elements and foreign modern design concepts, comprehend the aesthetic value of architectural art in Lingnan traditional architectural culture and the scientific value of Lingnan traditional architectural crafts, and then apply modern expression techniques to integrate the essence of Lingnan traditional elements into the advanced modern design concepts of foreign countries, so that different cultures can learn from each other and complement each other to achieve cultural blending.

For example, the Suzhou Museum designed by IM Pei is an excellent representative of the combination of Chinese and Western, reflecting the blending of different cultures. It is not only good at applying various traditional Chinese elements, such as traditional modeling elements, traditional gardening techniques, traditional ink paintings and traditional plants, but also good at using modern materials, simple and clear geometric shapes of modern art, perfect facilities and modern design concepts. The door of main entrance of the new Museum adopts the modeling elements of the traditional Chinese building (Fig.8). The two-door sloping beam structure is combined with the modern glass and metal materials to make the door have both traditional charm and the modern aesthetic concept.

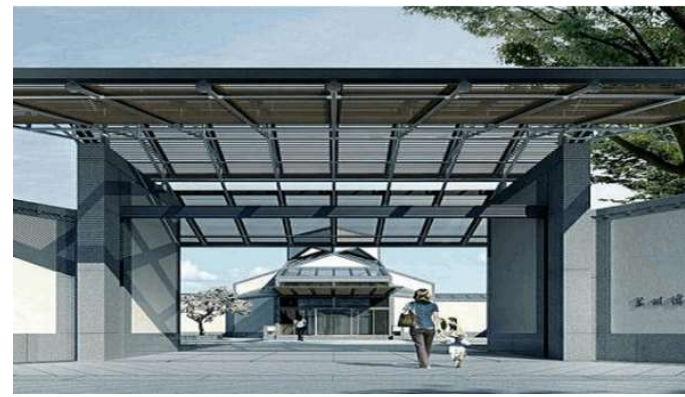

Figure 8. The door of the Suzhou Museum

\section{Conclusions}

In the architectural design, as a forward-looking architect, it is necessary to consciously use Lingnan traditional elements in our modern design, be good at applying modern design concepts and aesthetic concepts to transform traditional architectural elements, and creatively apply Lingnan traditional architectural elements so as to combine the traditional architectural elements of Lingnan with modern design concepts to create works with modern architectural designs and profound cultural heritage. Only in this way will our architectural design be able to have a permanent vitality, and then the characteristics rich in connotation will be revealed, avoid assimilation and superficiality.

\section{References}

1. [Britain] M. Gage, M. Vandenberg. Urban Hard Building Design [M]. Beijing: China Architecture and Building Press, 1985.

2. [U.S.] Motrock. Architectural Design Theory and Techniques [M]. translated by Li Jingyu, Li Shuo, $\mathrm{Wu}$ Xiuwei, 1st edition, 2007, Dalian: Dalian University of Technology Press.

3. Bai Na. The Influence of Chinese Traditional Culture on Garden Art [J]. Huge Stage, 2013, 12: 249-250.

4. Tumer T. City as landscape. Oxford: E\&FNSPon, 1996.

5. [Britain] Catherine Diin. The Architectural Form and Texture [M]. translated by Zhou Jianyun, Tang Xiaoxiang, Hou Yajuan, $1^{\text {st }}$ edition 2004, Hangzhou: Zhejiang Science and Technology Press.

6. Chen Ruilin. History of Chinese Modern Art Design [M]. Hunan: Hunan Science and Technology Press, $1^{\text {st }}$ edition: 131, 2002.

7. Ye Jundong. Discussion on the Innovative Application of Chinese Traditional Elements in Architectural Design [D]. Jiangsu: Suzhou University, 2011.

8. Zhou Guilin.Preliminary Study on the Application of Traditional Residential Decoration Elements in Contemporary Architectural Design [D]. North China University of Technology, 2012.

9. Wen Feifei. The Expression and Inheritance of Historical Context in Architectural Design --- Taking Xi'an Datang Furong Garden as an Example [D].Taiyuan: Taiyuan University of Technology, 2011 